\title{
Molecular Connections Between Circadian Clocks and Mood-related Behaviors
}

\author{
Urs Albrecht \\ Department of Biology, University of Fribourg, Fribourg, 1700, Switzerland
}

Correspondence to Urs Albrecht: urs.albrecht@unifr.ch

\begin{abstract}
The circadian system consists of individual cellular clocks. It organizes and synchronizes biochemical and physiological processes in order to optimally adapt an organism to its environment. This requires that the circadian system is responsive to environmental cues, which contain information about geophysical time (e.g., light), and allows an organism to predict daily recurring events. However, the system needs to be responsive to unpredictable cues (e.g., predators, stress) as well, which makes it vulnerable in its task to synchronize body functions on a 24-h time scale. If unpredictable signals occur only occasionally, this will have a minor effect on the clock system. Conversely, stress signals that occur more frequently will desynchronize the various cellular and tissue clocks in the body. This will result in biochemical and physiological disorder and as a consequence will lead to various diseases including neurological and mood disorders.

In this review, I will describe molecular mechanisms that have been associated with the circadian clock and mood-related behaviors.
\end{abstract}

\section{Introduction}

The circadian system is hierarchically organized and composed of cellular and tissue clocks. The various clocks in the cells of the peripheral and brain tissues are synchronized by a pacemaker located in the suprachiasmatic nuclei (SCN) in the ventral part of the hypothalamus [1]. Humoral, metabolic, and neural signals serve as messengers to synchronize the plethora of clocks in the body in order to obtain a coherent rhythmic organization of biological processes according to their necessity over the 24-h time scale. In order to trace seasonal changes over the year, environmental signals such as light, temperature, and humidity can influence the phase of body clocks and shift them to a very small extent every day. This assures that internal body time matches geophysical time allowing prediction of daily recurring events over the year.

If these signals, however, affect the organism at an unusual time as exemplified in traveling across time zones (jet lag), the organism's internal clock (having still the time of the origin of departure) becomes desynchronized relative to the day/night cycle of the arrival destination. Hence, the organism experiences light exposure during the subjective night and darkness during the subjective day. As a consequence, symptoms of irritability, malaise, diarrhea, and cognitive impairment are experienced [2]. Eventually, the organism's clock resynchronizes to the new environment due to the regular new light stimulus, glucocorticoid signaling, and other signals, such as melatonin and metabolic signals $[3,4]$. If environmental signals influence the organism in a random manner, as, for example, during stress experience, the circadian system is not able to resynchronize. This is associated with negative consequences on health, such as development of diabetes, obesity, cancer, and mood disorders.

At the molecular single cell level, the circadian clock can be described as an autoregulatory transcriptional/translational feedback loop involving a set of clock genes [5]. The main components encompass brain and muscle arnt-like factor (Bmal), circadian locomotor output cycles kaput (Clock), neuronal per-arnt-sim domain protein 2 (Npas2), period (Per), and cryptochrome (Cry). BMAL and CLOCK (or its paralog NPAS2) form a heterodimer 
and bind to specific sequences (E-boxes) in the promoter of clock (Per, Cry) and clock-controlled genes (ccg), thereby activating transcription of those genes. PER and CRY proteins form complexes that enter the nucleus to inhibit the activity of their own activators BMAL and CLOCK, thereby establishing an autoregulatory feedback loop. Activation and inhibition of the Bmal and Clock genes occur via the nuclear receptors Rev-erb (inhibition) and retinoid orphan receptor (Ror) (activation) in the so-called accessory loop, in which these nuclear receptors are also transcriptionally activated by BMAL and CLOCK.

Like at the systemic level, cellular clocks can respond to cell external cues, which can be among others, metabolites, hormones, and neurotransmitters. For example, light perceived by the intrinsically photosensitive retinal ganglion cells (ipRGCs) in the eye project directly via the retinohypothalamic tract $(\mathrm{RHT})$ to the SCN, where they release glutamate. This leads to activation of NMDA receptors in SCN neurons and subsequent activation of the CREB and calcium signaling pathways. This finally induces expression of immediate early genes including c-fos and the clock genes Per1 and to a lesser extent Per2. This initially leads to a shift of the phase of the circadian cycle in the cell and subsequently in the entire organism as observed in delayed or advanced onset of wheel running activity in rodents. Similar mechanisms are likely to occur in humans as well [6].

In the following, I will discuss how the earlier described basic mechanisms may impinge on the regulation of mood in rodents and whether this could be extended to humans.

\section{Evidence for the Involvement of the Clock in Mood-related Behaviors}

\section{Evidence in rodents}

Modeling depression and other mood-related behaviors in rodents is challenging due to the lack of biomarkers and the complex make up of moodrelated behaviors. Since motivation and despair are principal characteristics used to screen for moodrelated behaviors, particular tests are used, such as the forced swim test (FST), the tail suspension test (TST), and learned helplessness (LH). Anhedonia, another core symptom of depression, is assessed using the sucrose preference test or intracranial selfstimulation $[7,8]$. These paradigms are used in rodents in order to characterize the biological mechanisms associated with mood-related behaviors.

That clock genes may play a role in mood regulation is evidenced by their expression pattern in the brain areas of the reward system, which contributes to mood regulation. These brain areas include the ventral tegmental area (VTA), the nucleus accumbens (NAc), the amygdala (AMY), and the prefrontal cortex (PFC) [9]. Furthermore, mice carrying mutations in specific clock genes display alterations in mood-related behavioral tests described earlier. Mice with a mutation in the Clock gene exhibit a mania-like phenotype [10] and when treated with lithium, most behavioral responses are normalized to those of wild-type mice [11]. Mice with a mutation in the Per2 gene display a lower immobility time in the FST, altered neuronal activity, and higher dopamine levels in the striatum, correlating with changes in mood-related behavior [12]. Mice lacking Cry or Rev-erb $\alpha$ genes display altered behavior in several of the tests mentioned earlier [13-17], further supporting the evidence that the circadian clock directly and/or indirectly regulates mood-related behaviors in mice.

\section{Evidence in humans}

Early genomewide association studies of human gene polymorphisms have described correlations between circadian clock genes (such as Cry2, Clock, Npas2, and Bmal1) and various forms of depression [18-20]. These studies, however, were based on candidate gene approaches and appeared to be underpowered. Newer studies using further developed methodologies have failed to yield any association between human mood disorders and clock gene loci $[21,22]$. Despite these contradictory findings, it is established that treatments modulating the circadian clock (e.g., light therapy, sleep deprivation, lithium treatment) have positive effects on the relief of depressive symptoms [23], suggesting that the circadian system may have at least at the systemic level a regulatory influence on moodrelated behaviors in humans.

Why is it then that mouse studies show a much clearer relationship between the circadian clock and mood-related behaviors than human genetic studies?

One important reason may be found in the homogeneity of genetic background. In mouse studies, specific mouse strains with controls of the same genetic background are used with the consequence that there are lesser confounding variables in other genetic pathways that may influence mood-related behaviors. In human studies, however, the genetic background is more variable and "normal" versus "disease" cohorts show high variabilities and generate "genetic noise" that can cover small differences that may be significant. The sensitivity of patients with mood disorder to clock gene variants may be underpinned by changes in homeostatic metabolic mechanisms that regulate the circadian system [24], and these are highly variable in the human population. In order to address 
the genetic variability issue in humans, twin studies and familial studies have been performed $[25,26]$. They indicate that major depression results from genetic influences. However, environmental influences specific to an individual are also highly significant. These environmental factors are very difficult to control in humans (in contrast to laboratory mice). Hence, in order to unravel the molecular mechanisms between the circadian clock and moodrelated behaviors, the study of human subjects may provide correlative evidence at best. Comparison of human and mouse studies will therefore be synergistic combining the strength of both approaches, demonstration of relevance and molecular causality.

\section{Dopamine, Depression, and the Circadian Clock - Molecular Links}

A number of mouse studies have been performed highlighting molecular connections between the circadian clock machinery and the regulation of neurotransmitter synthesis and degradation. The best studied example so far is the regulation of dopamine metabolism. Interestingly, the rate-limiting enzymes for dopamine synthesis (tyrosine hydroxylase, TH) and degradation (monoamine oxidase, MAO) are both under circadian clock influence.

BMAL1 and NPAS2 could transcriptionally activate a luciferase reporter driven by the murine Maoa promoter in a circadian fashion. Interestingly, the BMAL1 protein was recruited to the Maoa promoter in the brain tissue as evidenced by chromatin immunoprecipitation, indicating that Maoa is directly regulated by clock components [12]. Unexpectedly, the PER2 protein modulated the BMAL1/NPAS2driven activation in a positive fashion, leading to increased Maoa mRNA levels. This is in contrast to its negative role on its own transcription in the SCN. It appears that PER2 protein function depends strongly on the cellular context and relative amounts of other clock and nonclock proteins [27]. However, consistent with the positive role of PER2 in the BMAL1/NPAS2-dependent transcription of Maoa, mice with a mutation in the Per2 gene (in frame deletion of 87 amino acids at the end of the PAS domain [28]) showed decreased Maoa mRNA and protein levels. Hence, dopamine degradation was reduced in Per2 mutant mice, and dopamine levels in the striatum were increased as measured by microdialysis in living mice. This was paralleled by a mania-like phenotype and changes in neuronal activity in response to MAO inhibitors [12]. Taken together, these observations indicated that dopamine degradation is modulated by the circadian clock.

Dopamine synthesis also appears to be under clock influence. TH converts tyrosine into dihydroxyphenylalanine (DOPA), which then is converted by

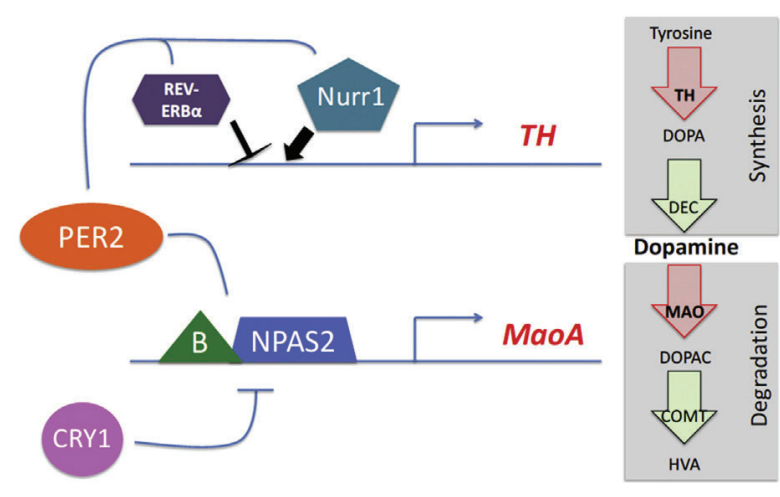

Fig. 1. Influence of clock components on transcriptional activation of rate-limiting enzymes (red) in dopamine metabolism. Top: Synthesis of dopamine from tyrosine is gated by tyrosine hydroxylase $(\mathrm{TH})$. This enzyme is regulated by the nuclear receptors REV-ERB $\alpha$ and Nurr1, which repress or activate $T H$ transcription, respectively. PER2 has the potential to interact with both of them, thereby derepressing and/or coactivating $\mathrm{TH}$ transcription. Bottom: Degradation of dopamine is gated by monoamine oxidase (MAO). Transcription of this enzyme is regulated by the clock components BMAL1 (B) and NPAS2. PER2 increases their activity through interaction with the BMAL1/ NPAS2 complex, whereas CRY1 suppresses the activity of the BMAL1/NPAS2 complex.

DOPA decarboxylase (DEC) into dopamine (Fig. 1). In this synthesis, the rate-limiting enzyme is $\mathrm{TH}$, which shows in its promoter elements for nuclear receptor binding. Chung et al. (2014) showed that REV-ERB $\alpha$ (a clock component) repressed the Th promoter, whereas retinoic orphan receptor $\alpha$ $(\mathrm{ROR} \alpha)$ and nuclear-receptor-related protein 1 (NURR1) activated it [13]. Chromatin immunoprecipitation revealed that REV-ERB $\alpha$ and NURR 1 were binding to the Th promoter in an antagonistic manner (Fig. 1). Accordingly, Rev-erb $\alpha$ knock-out mice displayed elevated levels of Th mRNA and protein leading to increased dopamine amounts and firing rate in the striatum [13]. Consistent with these observations, these animals showed mania-like behavior characterized by decreased anxiety, hyperactivity, increased risk-taking, and augmented aggression [13,15-17]. Because PER2 was shown to interact with nuclear receptors, including REV$\mathrm{ERB} \alpha$ and NURR1 [29], it can be hypothesized that interactions with PER2 at the protein level may allow temporal synchronization of action of these two nuclear receptors.

Dopamine binds to a family of receptors termed dopamine receptors $(D r d)$. Drd3 mRNA displayed a diurnal profile in the striatum [30], and mice with a mutation in Drd3 exhibited increased sensitivity to concurrent stimulation of DRD1 and DRD2 leading to altered emotional and depressive behavior in mice [31]. Ikeda et al. (2013) provided evidence that REV$\mathrm{ERB} \alpha$ and ROR $\alpha$ regulate the expression of $\mathrm{Drd} 3$ 
[32]. This may be important to synchronize Drd3 expression with dopamine production in the striatum in order to restrict dopamine signaling to a particular time window. This suggests that targeting DRD3 and/or REV-ERB $\alpha$ and ROR $\alpha$ pharmacologically should profit from timed application, which would reduce dosage and diminish side effects, such as weight gain, which is frequently observed in patients treated for mood disorders.

The dopaminergic system is composed of two main pathways, the mesolimbic and the nigrostriatal pathways. While the mesolimbic pathway is important for the control of motivation, emotion, and reward functions [33], the nigrostriatal pathway is associated with the regulation of motor function and to some extent reward [34]. Abnormalities in the mesolimbic pathway are associated with addiction, affective disorders, schizophrenia, and attentiondeficit hyperactivity disorder [35-37], while the degeneration of the nigrostriatal pathway can induce Parkinson's disease [38], which may be associated with some degree of depression $[39,40]$. Interestingly, expression of clock genes is oscillating in a circadian manner in the substantia nigra (SN, nigrostriatal pathway) and the VTA (mesolimbic pathway) $[9,13]$ with $\mathrm{TH}$ showing also a time of day-dependent expression pattern [13,41,42]. Taken together, the current data suggest that the dopaminergic systems are under circadian clock influence.

\section{Molecular Relationships Between the HPA Axis, Mood Disorders, and the Circadian Clock}

The hypothalamic-pituitary-adrenal (HPA) axis and glucocorticoid dysregulation have been implicated in mood disorders [43]. Hypercortisolism was observed in patients with depression [44-46], while hypocortisolism was seen in atypical depression characterized by symptoms such as lethargy, fatigue, hyperphagia, and weight gain [47].

Glucocorticoid levels show a robust daily rhythm of expression in mice and men, with peak levels occurring at the beginning of the active phase. This temporal rhythmicity is controlled by diurnal variation in corticotropin-releasing factor (CRF) and adrenocorticotropic hormone (ACTH) activity, as well as the peripheral clock in the adrenal glands [48-50]. Since glucocorticoids act via binding to the glucocorticoid receptor (GR), it is important to note that conditional mutagenesis of GR in the nervous system of mice affected the emotional state of these animals [51]. Furthermore, overexpression of GR led to depression-like behavior, which was accompanied by an enhancement of sensitization to cocaine [52]. This was consistent with the observation that GR may be a potential target to reduce cocaine abuse [53].
Those findings illustrate the involvement of GR in mood-related behaviors.

Although the amount of glucocorticoids displayed a circadian rhythm in the serum, the GR receptor was expressed in a more or less constant manner over the $24 \mathrm{~h}$ in many tissues (reviewed in Ref. [54]). Hence, rhythmic expression of GR target genes may be influenced by GR's rhythmic ligand. Binding of the ligand leads to nuclear translocation of nuclear receptors. This is the case for GR as well. However, this process seemed also to be coordinated by another nuclear receptor, the circadian clock component REV-ERB $\alpha$. In the liver, REV-ERB $\alpha$ competed with GR for heat-shock protein (HSP) 90 binding, and thereby these two nuclear receptors influenced each other's stability and nuclear localization in an opposite manner (Fig. 2). This was paralleled by opposite rhythmicity of REV-ERB $\alpha$ and GR target gene expression $[55,56]$. It remains to be seen, however, whether this mechanism is also operative in the brain tissue. As described earlier, mood-related behavior and dopamine metabolism were altered in Rev-erb $\alpha$ knock-out mice. This may also involve GR, because it regulates catechol-Omethyltransferase (Comt) [55], an enzyme degrading the MAOA product 3,4-dihydroxyphenylacetic acid (DOPAC) to homovanillic acid (HVA) (Fig. 1). Therefore, it is likely that the monoaminergic system, the glucocorticoid pathway, and the circadian clock are linked via GR and REV-ERB $\alpha$.

In mouse liver, GR was also modulated by cryptochrome (Cry) proteins in a ligand-dependent fashion leading to rhythmic repression of GR activity and suppression of the HPA axis [57]. Mice lacking

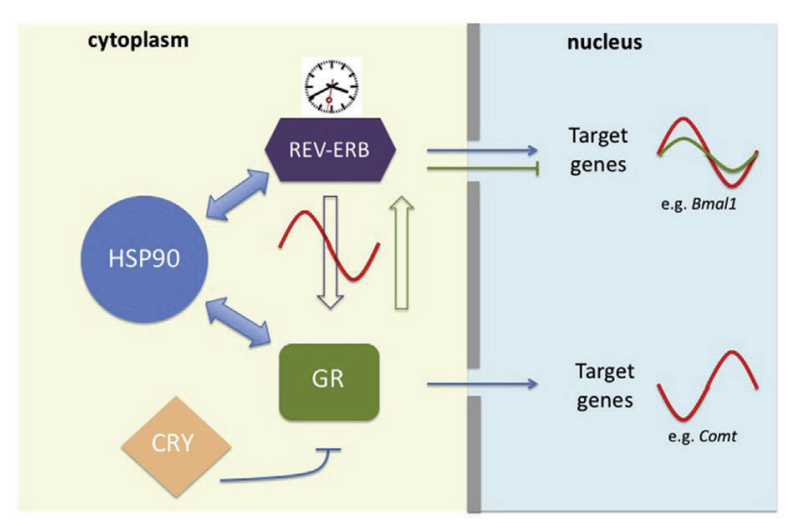

Fig. 2. Model of reciprocal influence of REV-ERB $\alpha$ and glucocorticoid receptor (GR) on each other's nuclear/ cytoplasmic distribution. The two receptors can bind to HSP90, which binds either REV-ERB $\alpha$ or GR at a given time. The amount of REV-ERB $\alpha$ is clock regulated and hence submits GR to an inverse rhythmic cellular distribution. As a consequence, the target genes of REV-ERB $\alpha$ and GR display opposite circadian phase of transcription. Additionally, GR can be repressed by Cry. 
Cry1 displayed reduced levels of dopamine in the striatum and showed depression-like behavior $[14,15]$. Since Cry proteins are suppressing not only GR activity (Fig. 2) but also BMAL1/NPAS2 activity (Fig. 1), Cry appears to act on the system of mood regulation at multiple levels.

CHRONO, another repressor in the circadian clock mechanism, appeared to affect the HPA axis in a similar manner as Cry [58]. However, it is unknown whether Chrono knock-out mice show alterations in mood-related behaviors. Overall it appears that clock components gate glucocorticoid signaling in order to establish circadian signals related to predictable "stress" factors, such as light-dark changes, and energy requirements. However, bursts of glucocorticoids secreted into the blood stream in response to unexpected stress factors, such as unexpected emergence of a predator, can override the circadian system and secure an organism's survival. In contrast, overriding the circadian system permanently will lead to disorganization of biochemical pathways, including, for example, ordered dopamine metabolism, and lay the foundation for the development of mood disorders and other neurological diseases.

\section{Light Affects Brain Function}

Light at night has strong effects on physiology in mice and humans. It suppresses melatonin and disrupts glucocorticoid signaling and attenuates circadian clock function. As a consequence, sleep is impaired, body weight regulation is abnormal, and brain functions including mood regulation are altered (reviewed in Refs. [59,60]).

As described earlier, light is perceived by the retina of the eye via specialized cells, the ipRGCs. Light is transformed into an electrochemical signal and is conducted via the RHT to the SCN and other brain nuclei [61] (Fig. 3). Interestingly, genetic ablation of ipRGCs in mice abrogated light-signal reception at the SCN and resetting of the circadian clock was affected as well as other brain functions [62]. Notably, lack of ipRGCs in mice impacted on lightinduced mood regulation and learning $[63,64]$ indicating that ipRGCs were important for driving these light-mediated behavioral effects. It appeared that distinct subpopulations of ipRGCs drive the effects on learning and mood. ipRGCs that project to the SCN affected learning without disrupting the clock in the SCN. Another set of ipRGCs projects to the perihabenula (PHb) (Fig. 3) and regulates moodrelated behaviors, which suggested that the $\mathrm{PHb}$ is integrated into a distinctive circuitry with mood regulating centers [65].

Support for the involvement of light in mood regulation comes from clinical studies in humans that indicate an antidepressive effect of light therapy

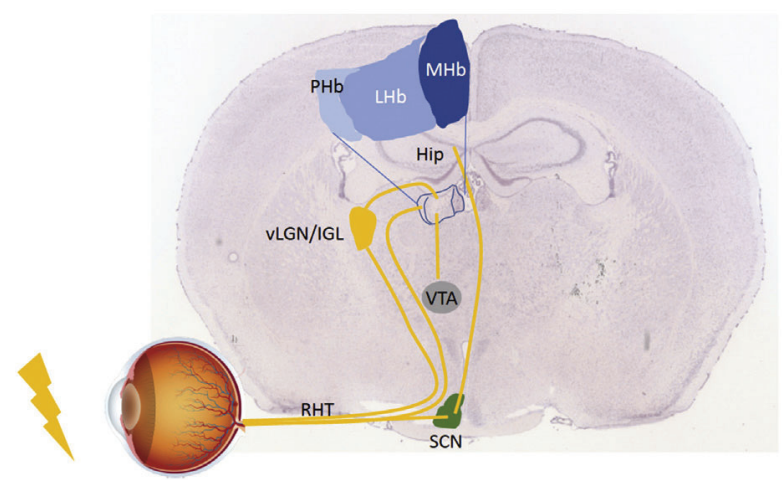

Fig. 3. Model of light input into the brain. Shown is a coronal mouse brain section with various brain nuclei highlighted in color. The habenula is enlarged to show the subdivisions of this region (blue). Light is perceived by the retina of the eye and transformed into an electrochemical signal, which is transporter via the retinohypothalamic tract (RHT) to various brain nuclei, including the SCN (green), $\mathrm{PHb}$ (light blue) and the vLGN/IGL (yellow). From the SCN (green), this signal reaches the hippocampus (Hip) and influences memory. From the vLGN/IGL (yellow), the signal reaches the $\mathrm{LHb}$ (medium blue) from where it affects the VTA (grey) ultimately influencing mood-related behaviors. The light signal reaching the $\mathrm{PHb}$ directly from the retina also affects the VTA and influences mood.

[66-72]. The circuit related to light therapy has recently been investigated in rodents. Light could influence activity of neurons in the lateral habenula $(\mathrm{LHb})$, which was accompanied by changes in depressive-like behaviors [64,73]. Combining neurotracer and transneuronal virus tracing techniques, Huang et al. (2019) could show that in mice a disynaptic visual circuit connected the retina and the $\mathrm{LHb}$ via the ventral lateral geniculate nucleus and intergeniculate leaflet (vLGN/IGL) (Fig. 3). This disynaptic retina-vLGN/IGL-LHb pathway appeared to underlie the antidepressive effects of light therapy in mice [74]. Given the high conservation of ipRGCs and visual thalamus to habenula projection in rodents and humans, the retina-vLGN/IGL-LHb pathway is most likely involved in the effects of light therapy in humans as well. However, how the light input to the habenula affects mood-related behaviors at the molecular level remains elusive.

\section{Future Questions}

The circadian clock reciprocally interacts with many physiologically relevant systems and processes in the brain and peripheral tissues, including monoaminergic and glutamatergic signaling, HPA axis function, metabolism, and immune function [75]. Given this multitude of interactions, the circadian clock most likely influences mood-related behaviors through many avenues. At the systemic level, 
several relationships have been established; however, the molecular mechanisms governing these relationships are still mostly unclear. Although we know that light can influence in addition to the SCN other brain regions, it is entirely unclear how this is mechanistically related to the monoaminergic, glutamatergic, and adrenocortical signaling systems. Future studies will have to be directed not only toward the anatomical relationships between brain nuclei relevant for mood regulation but also toward the dynamic influence of these brain regions on each other's gene expression. The difficulty for such an approach is that a "snapshot" at a specific time will most likely not reveal the molecular relationships between the nuclei of the network regulating mood. With the current high-throughput techniques, however, it should be possible to generate temporal maps of gene expression profiles in response to various stimuli, including light. This will provide a framework for starting hypothesisdriven experiments investigating molecular mechanisms regulating mood at multiple levels.

\section{Acknowledgments}

I would like to thank Dr. Jürgen Ripperger for comments on the manuscript. The support by the Velux Foundation and the Swiss National Science Foundation is gratefully acknowledged.

Received 5 November 2019; Received in revised form 26 November 2019; Accepted 26 November 2019 Available online 18 December 2019

Keywords: HPA axis; glucocorticoids; dopamine; nuclear receptors; light

\footnotetext{
Abbreviations used:

ACTH, adrenocorticotropic hormone; AMY, amygdala; BMAL, brain and muscle arnt-like factor; CLOCK, circadian locomotor output cycles kaput; CRY, cryptochrome; CRF, corticotropin-releasing factor; DEC, DOPA decarboxylase; DOPA, dihydroxyphenylalanine; DOPAC, dihydroxyphenylacetic acid; DRD, dopamine receptor; FST,

forced swim test; GR, glucocorticoid receptor; HPA, hypothalamic-pituitary-adrenal; HSP, heat-shock protein; HVA, homovanillic acid; ipRGCs, intrinsically photosensitive retinal ganglion cells; $\mathrm{LH}$, learned helplessness; $\mathrm{LHb}$, lateral habenula; MAO, monoamine oxidase; NAc, nucleus accumbens; NPAS2, neuronal per-arnt-sim domain protein 2; NURR1, nuclear-receptor-related protein
}

1; PER, period; PFC, prefrontal cortex; $\mathrm{PHb}$, perihabenula; $\mathrm{RHT}$, retinohypothalamic tract; ROR, retinoid orphan receptor; SCN, suprachiasmatic nuclei; SN, substantia nigra; $\mathrm{TH}$, tyrosine hydroxylase; TST, tail suspension test; vLGN/IGL, ventral lateral geniculate nucleus and intergeniculate leaflet; VTA, ventral tegmental area.

\section{References}

[1] C. Dibner, U. Schibler, U. Albrecht, The mammalian circadian timing system: organization and coordination of central and peripheral clocks, Annu. Rev. Physiol. 72 (2010) 517-549.

[2] R.G. Foster, L. Kreitzman, The rhythms of life: what your body clock means to you!, Exp. Physiol. 99 (2014) 599-606.

[3] J.A. Mohawk, K. Cashen, T.M. Lee, Inhibiting cortisol response accelerates recovery from a photic phase shift, Am. J. Physiol. Regul. Integr. Comp. Physiol. 288 (2005) R221-R228.

[4] S. Kiessling, G. Eichele, H. Oster, Adrenal glucocorticoids have a key role in circadian resynchronization in a mouse model of jet lag, J. Clin. Investig. 120 (2010) 2600-2609.

[5] J.S. Takahashi, Transcriptional architecture of the mammalian circadian clock, Nat. Rev. Genet. 18 (2017) 164-179.

[6] C. Cajochen, C. Jud, M. Munch, S. Kobialka, A. Wirz-Justice, $\mathrm{U}$. Albrecht, Evening exposure to blue light stimulates the expression of the clock gene PER2 in humans, Eur. J. Neurosci. 23 (2006) 1082-1086.

[7] J.N. Crawley, What's Wrong With My Mouse? Behavioral Phenotyping of Transgenic and Knock-Out Mice, Wiley-Liss., New York, 2000.

[8] D.D. Pollak, C.E. Rey, F.J. Monje, Rodent models in depression research: classical strategies and new directions, Ann. Med. 42 (2010) 252-264.

[9] C. Guilding, H.D. Piggins, Challenging the omnipotence of the suprachiasmatic timekeeper: are circadian oscillators present throughout the mammalian brain? Eur. J. Neurosci. 25 (2007) 3195-3216.

[10] C.A. McClung, K. Sidiropoulou, M. Vitaterna, J.S. Takahashi, F.J. White, D.C. Cooper, et al., Regulation of dopaminergic transmission and cocaine reward by the Clock gene, Proc. Natl. Acad. Sci. U. S. A. 102 (2005) 9377-9381.

[11] K. Roybal, D. Theobold, A. Graham, J.A. DiNieri, S.J. Russo, V. Krishnan, et al., Mania-like behavior induced by disruption of CLOCK, Proc. Natl. Acad. Sci. U. S. A. 104 (2007) 6406-6411.

[12] G. Hampp, J.A. Ripperger, T. Houben, I. Schmutz, C. Blex, S. Perreau-Lenz, et al., Regulation of monoamine oxidase A by circadian-clock components implies clock influence on mood, Curr. Biol. 18 (2008) 678-683.

[13] S. Chung, E.J. Lee, S. Yun, H.K. Choe, S.B. Park, H.J. Son, et al., Impact of circadian nuclear receptor REV-ERBalpha on midbrain dopamine production and mood regulation, Cell 157 (2014) 858-868.

[14] D. De Bundel, G. Gangarossa, A. Biever, X. Bonnefont, E. Valjent, Cognitive dysfunction, elevated anxiety, and reduced cocaine response in circadian clock-deficient cryptochrome knockout mice, Front. Behav. Neurosci. 7 (2013) 152. 
[15] A. Schnell, F. Sandrelli, V. Ranc, J.A. Ripperger, E. Brai, L. Alberi, et al., Mice lacking circadian clock components display different mood-related behaviors and do not respond uniformly to chronic lithium treatment, Chronobiol. Int. 32 (2015) 1075-1089.

[16] A. Schnell, S. Chappuis, I. Schmutz, E. Brai, J.A. Ripperger, et al., The nuclear receptor REV-ERB $\alpha$ regulates Fabp7 and modulates adult hippocampal neurogenesis, PLoS One 9 (2014), e99883.

[17] J. Jager, W.T. O'Brien, J. Manlove, E.N. Krizman, B. Fang, et al., Behavioral changes and dopaminergic dysregulation in mice lacking the nuclear receptor Rev-erb alpha, Mol. Endocrinol. 28 (2014) 490-498.

[18] H.A. Mansour, J. Wood, T. Logue, K.V. Chowdari, M. Dayal, D.J. Kupfer, et al., Association study of eight circadian genes with bipolar I disorder, schizoaffective disorder and schizophrenia, Genes Brain Behav. 5 (2006) 150-157.

[19] C. Lavebratt, L.K. Sjoholm, P. Soronen, T. Paunio, M.P. Vawter, W.E. Bunney, et al., CRY2 is associated with depression, PLoS One 5 (2010), e9407.

[20] M.J. McCarthy, C.M. Nievergelt, J.R. Kelsoe, D.K. Welsh, A survey of genomic studies supports association of circadian clock genes with bipolar disorder spectrum illnesses and lithium response, PLoS One 7 (2012), e32091.

[21] C.L. Hyde, M.W. Nagle, C. Tian, X. Chen, S.A. Paciga, J.R. Wendland, et al., Identification of 15 genetic loci associated with risk of major depression in individuals of European descent, Nat. Genet. 48 (2016) 1031-1036.

[22] CONVERGE Consortium, Sparse whole-genome sequencing identifies two loci for major depressive disorder, Nature 523 (2015) 588-591.

[23] A. Wirz-Justice, F. Benedetti, Perspectives in affective disorders: clocks and sleep, Eur. J. Neurosci. (2019 Jan 31), https://doi.org/10.1111/ejn.14362 [Epub ahead of print] Review. PMID: 30702783.

[24] C. Garbazza, F. Benedetti, Genetic factors affecting seasonality, mood, and the circadian clock, Front. Endocrinol. 9 (2018) 481.

[25] P.F. Sullivan, M.C. Neale, K.S. Kendler, Genetic epidemiology of major depression: review and meta-analysis, Am. J. Psychiatry 157 (2000) 1552-1562.

[26] A. Starnawska, Q. Tan, M. Soerensen, M. McGue, O. Mors, et al., Epigenome-wide association study of depression symptomatology in elderly monozygotic twins, Transl. Psychiatry 9 (2019) 214.

[27] Y. Ogawa, N. Koike, G. Kurosawa, T. Soga, M. Tomita, $\mathrm{H}$. Tei, Positive autoregulation delays the expression phase of mammalian clock gene Per2, PLoS One 6 (2011), e18663.

[28] B. Zheng, D.W. Larkin, U. Albrecht, Z.S. Sun, M. Sage, G. Eichele, et al., The mPer2 gene encodes a functional component of the mammalian circadian clock, Nature 400 (1999) 169-173.

[29] I. Schmutz, J.A. Ripperger, S. Baeriswyl-Aebischer, U. Albrecht, The mammalian clock component PERIOD2 coordinates circadian output by interaction with nuclear receptors, Genes Dev. 24 (2010) 345-357.

[30] M. Akhisaroglu, M. Kurtuncu, H. Manev, T. Uz, Diurnal rhythms in quinpirole-induced locomotor behaviors and striatal D2/D3 receptor levels in mice, Pharmacol. Biochem. Behav. 80 (2005) 371-377.

[31] M. Xu, T.E. Koeltzow, G.T. Santiago, R. Moratalla, D.C. Cooper, X.T. Hu, et al., Dopamine D3 receptor mutant mice exhibit increased behavioral sensitivity to concurrent stimulation of D1 and D2 receptors, Neuron 19 (1997) 837-848.

[32] E. Ikeda, N. Matsunaga, K. Kakimoto, K. Hamamura, A. Hayashi, S. Koyanagi, et al., Molecular mechanism regulating 24-hour rhythm of dopamine D3 receptor expression in mouse ventral striatum, Mol. Pharmacol. 83 (2013) 959-967.

[33] E.J. Nestler, W.A. Carlezon Jr., The mesolimbic dopamine reward circuit in depression, Biol. Psychiatry 59 (2006) 1151-1159.

[34] R.A. Wise, Drug-activation of brain reward pathways, Drug Alcohol Depend. 51 (1998) 13-22.

[35] A.A. Grace, Dysregulation of the dopamine system in the pathophysiology of schizophrenia and depression, Nat. Rev. Neurosci. 17 (2016) 524-532.

[36] S.E. Hyman, R.C. Malenka, Addiction and the brain: the neurobiology of compulsion and its persistence, Nat. Rev. Neurosci. 2 (2001) 695-703.

[37] N.D. Volkow, G.J. Wang, S.H. Kollins, T.L. Wigal, J.H. Newcorn, F. Telang, et al., Evaluating dopamine reward pathway in ADHD: clinical implications, JAMA 302 (2009) 1084-1091.

[38] H.C. Cheng, C.M. Ulane, R.E. Burke, Clinical progression in Parkinson disease and the neurobiology of axons, Ann. Neurol. 67 (2010) 715-725.

[39] D.J. Burn, Beyond the iron mask: towards better recognition and treatment of depression associated with Parkinson's disease, Mov. Disord.: official journal of the Movement Disorder Society 17 (2002) 445-454.

[40] K.R. Chaudhuri, A.H. Schapira, Non-motor symptoms of Parkinson's disease: dopaminergic pathophysiology and treatment, Lancet Neurol. 8 (2009) 464-474.

[41] M. Weber, T. Lauterburg, I. Tobler, J.M. Burgunder, Circadian patterns of neurotransmitter related gene expression in motor regions of the rat brain, Neurosci. Lett. 358 (2004) $17-20$.

[42] I.C. Webb, R.M. Baltazar, X. Wang, K.K. Pitchers, L.M. Coolen, M.N. Lehman, Diurnal variations in natural and drug reward, mesolimbic tyrosine hydroxylase, and clock gene expression in the male rat, J. Biol. Rhythm. 24 (2009) 465-476.

[43] C.M. Pariante, S.L. Lightman, The HPA axis in major depression: classical theories and new developments, Trends Neurosci. 31 (2008) 464-468.

[44] K. Hinkelmann, S. Moritz, J. Botzenhardt, C. Muhtz, K. Wiedemann, M. Kellner, et al., Changes in cortisol secretion during antidepressive treatment and cognitive improvement in patients with major depression: a longitudinal study, Psychoneuroendocrinology 37 (2012) 685-692.

[45] R.T. Rubin, R.E. Poland, I.M. Lesser, R.A. Winston, A.L. Blodgett, Neuroendocrine aspects of primary endogenous depression. I. Cortisol secretory dynamics in patients and matched controls, Arch. Gen. Psychiatr. 44 (1987) 328-336.

[46] P. Linkowski, J. Mendlewicz, M. Kerkhofs, R. Leclercq, J. Golstein, M. Brasseur, et al., 24-hour profiles of adrenocorticotropin, cortisol, and growth hormone in major depressive illness: effect of antidepressant treatment, J. Clin. Endocrinol. Metab. 65 (1987) 141-152.

[47] P.W. Gold, G.P. Chrousos, Organization of the stress system and its dysregulation in melancholic and atypical depression: high vs low CRH/NE states, Mol. Psychiatry 7 (2002) 254-275. 
[48] A. Ishida, T. Mutoh, T. Ueyama, H. Bando, S. Masubuchi, D. Nakahara, et al., Light activates the adrenal gland: timing of gene expression and glucocorticoid release, Cell Metabol. 2 (2005) 297-307.

[49] N. Nader, G.P. Chrousos, T. Kino, Interactions of the circadian CLOCK system and the HPA axis, Trends Endocrinol. Metab.: TEM (Trends Endocrinol. Metab.) 21 (2010) 277-286.

[50] H. Oster, S. Damerow, S. Kiessling, V. Jakubcakova, D. Abraham, J. Tian, et al., The circadian rhythm of glucocorticoids is regulated by a gating mechanism residing in the adrenal cortical clock, Cell Metabol. 4 (2006) 163-173.

[51] F. Tronche, C. Kellendonk, O. Kretz, P. Gass, K. Anlag, P.C. Orban, et al., Disruption of the glucocorticoid receptor gene in the nervous system results in reduced anxiety, Nat. Genet. 23 (1999) 99-103.

[52] Q. Wei, X.Y. Lu, L. Liu, G. Schafer, K.R. Shieh, S. Burke, et al., Glucocorticoid receptor overexpression in forebrain: a mouse model of increased emotional lability, Proc. Natl. Acad. Sci. U. S. A. 101 (2004) 11851-11856.

[53] V. Deroche-Gamonet, I. Sillaber, B. Aouizerate, R. Izawa, M. Jaber, S. Ghozland, et al., The glucocorticoid receptor as a potential target to reduce cocaine abuse, J. Neurosci. 23 (2003) 4785-4790.

[54] H. Oster, E. Challet, V. Ott, E. Arvat, E.R. de Kloet, D.J. Dijk, et al., The functional and clinical significance of the 24-hour rhythm of circulating glucocorticoids, Endocr. Rev. 38 (2017) 3-45.

[55] T. Okabe, R. Chavan, S.S. Fonseca Costa, A. Brenna, J.A. Ripperger, U. Albrecht, REV-ERBalpha influences the stability and nuclear localization of the glucocorticoid receptor, J. Cell Sci. 129 (2016) 4143-4154.

[56] G. Caratti, M. Iqbal, L. Hunter, D. Kim, P. Wang, R.M. Vonslow, et al., REVERBa couples the circadian clock to hepatic glucocorticoid action, J. Clin. Investig. 128 (2018) 4454-4471.

[57] K.A. Lamia, S.J. Papp, R.T. Yu, G.D. Barish, N.H. Uhlenhaut, J.W. Jonker, et al., Cryptochromes mediate rhythmic repression of the glucocorticoid receptor, Nature 480 (2011) 552-556.

[58] A. Goriki, F. Hatanaka, J. Myung, J.K. Kim, T. Yoritaka, S. Tanoue, et al., A novel protein, CHRONO, functions as a core component of the mammalian circadian clock, PLoS Biol. 12 (2014), e1001839.

[59] L.K. Fonken, R.J. Nelson, The effects of light at night on circadian clocks and metabolism, Endocr. Rev. 35 (2014) 648-670.

[60] T.A. Bedrosian, R.J. Nelson, Timing of light exposure affects mood and brain circuits, Transl. Psychiatry 7 (2017), e1017.

[61] S. Hattar, M. Kumar, A. Park, P. Tong, J. Tung, K.W. Yau, et al., Central projections of melanopsin-expressing retinal ganglion cells in the mouse, J. Comp. Neurol. 497 (2006) 326-349.

[62] A.D. Guler, J.L. Ecker, G.S. Lall, S. Haq, C.M. Altimus, H.W. Liao, et al., Melanopsin cells are the principal conduits for rod-cone input to non-image-forming vision, Nature 453 (2008) 102-105.

[63] T.A. LeGates, C.M. Altimus, H. Wang, H.K. Lee, S. Yang, H. Zhao, et al., Aberrant light directly impairs mood and learning through melanopsin-expressing neurons, Nature 491 (2012) 594-598.

[64] T.A. LeGates, D.C. Fernandez, S. Hattar, Light as a central modulator of circadian rhythms, sleep and affect, Nat. Rev. Neurosci. 15 (2014) 443-454.

[65] D.C. Fernandez, P.M. Fogerson, L. Lazzerini Ospri, M.B. Thomsen, R.M. Layne, D. Severin, et al., Light affects mood and learning through distinct retina-brain pathways, Cell 175 (2018) 71-84 e18.

[66] N.E. Rosenthal, D.A. Sack, J.C. Gillin, A.J. Lewy, F.K. Goodwin, Y. Davenport, et al., Seasonal affective disorder. A description of the syndrome and preliminary findings with light therapy, Arch. Gen. Psychiatr. 41 (1984) $72-80$.

[67] D.F. Kripke, Light treatment for nonseasonal depression: speed, efficacy, and combined treatment, J. Affect. Disord. 49 (1998) 109-117.

[68] R.N. Golden, B.N. Gaynes, R.D. Ekstrom, R.M. Hamer, F.M. Jacobsen, T. Suppes, et al., The efficacy of light therapy in the treatment of mood disorders: a review and meta-analysis of the evidence, Am. J. Psychiatry 162 (2005) 656-662.

[69] A. Wirz-Justice, F. Benedetti, M. Terman, Chronotherapeutics for Affective Disorders, second ed. ed, Karger, Basel, 2013.

[70] R. Lieverse, E.J. Van Someren, M.M. Nielen, B.M. Uitdehaag, J.H. Smit, W.J. Hoogendijk, Bright light treatment in elderly patients with nonseasonal major depressive disorder: a randomized placebo-controlled trial, Arch. Gen. Psychiatr. 68 (2011) 61-70.

[71] R.W. Lam, A.J. Levitt, R.D. Levitan, E.E. Michalak, A.H. Cheung, R. Morehouse, et al., Efficacy of bright light treatment, fluoxetine, and the combination in patients with nonseasonal major depressive disorder: a randomized clinical trial, JAMA Psychiatr. 73 (2016) 56-63.

[72] D.K. Sit, J. McGowan, C. Wiltrout, R.S. Diler, J.J. Dills, J. Luther, et al., Adjunctive bright light therapy for bipolar depression: a randomized double-blind placebo-controlled trial, Am. J. Psychiatry 175 (2018) 131-139.

[73] H. Zhao, B. Rusak, Circadian firing-rate rhythms and light responses of rat habenular nucleus neurons in vivo and in vitro, Neuroscience 132 (2005) 519-528.

[74] L. Huang, Y. Xi, Y. Peng, Y. Yang, X. Huang, Y. Fu, et al., A visual circuit related to habenula underlies the antidepressive effects of light therapy, Neuron 102 (2019) 128-142 e8.

[75] K.D. Ketchesin, D. Becker-Krail, C.A. McClung, Moodrelated central and peripheral clocks, Eur. J. Neurosci. (2018) 1-20, https://doi.org/10.1111/ejn.14253. 